(2)

\title{
Thiazolidinedione-induced lipid droplet formation during osteogenic differentiation
}

\author{
M van de Vyver", E Andrag ${ }^{*}$, I L Cockburn and W F Ferris
}

Division of Endocrinology, Department of Medicine, Faculty of Medicine and Health Sciences, Stellenbosch University, PO Box 19063, Tygerberg 7505, South Africa

*(M van de Vyver and $\mathrm{E}$ Andrag contributed equally to this work)

Correspondence should be addressed to W F Ferris

Email wferris@sun.ac.za

\begin{abstract}
Chronic administration of the insulin-sensitising drugs, thiazolidinediones (TZDs), results in low bone mineral density and 'fatty bones'. This is thought to be due, at least in part, to aberrant differentiation of progenitor mesenchymal stem cells (MSCs) away from osteogenesis towards adipogenesis. This study directly compared the effects of rosiglitazone, pioglitazone, and netoglitazone treatment on osteogenesis and adipogenesis in MSCs derived from subcutaneous (SC) or visceral (PV) white adipose tissue. MSCs were isolated from adipose tissue depots of male Wistar rats and characterised using flow cytometry. The effects of TZD treatment on osteogenic and adipogenic differentiation were assessed histologically (day 14) and by quantitative PCR analysis (Ppary2 (Pparg2), Ap2 (Fabp4), Adipsin (Adps), Msx2, Collagen I (Col1a1), and Alp) on days 0,7 , and 10. Uniquely, lipid droplet formation and mineralisation were found to occur concurrently in response to TZD treatment during osteogenesis. Compared with SC MSCs, PV MSCs were more prone to lipid accumulation under controlled osteogenic and adipogenic differentiation conditions. This study demonstrated that the extent of lipid accumulation is dependent on the nature of the Ppar ligand and that SC and PV MSCs respond differently to in vitro TZD treatment, suggesting that metabolic status can contribute to the adverse effects associated with TZD treatment.
\end{abstract}

\author{
Key Words \\ - rosiglitazone \\ - netoglitazone \\ - pioglitazone \\ - mesenchymal stem cells
}

\section{Introduction}

Thiazolidinediones (TZDs; also known as glitazones) have a unique insulin-sensitising action (Bergeron et al. 2006); however, the clinical use of TZDs for the treatment of type 2 diabetes mellitus is currently challenged by reports of an inadequate safety profile (Cariou et al. 2012). In addition to body weight gain (van Dieren et al. 2012), fluid retention (Karalliedde et al. 2006), congestive heart failure (Gitt et al. 2012), and cancer (Mamtani et al. 2012), the chronic administration of TZDs may result in low bone mineral density and increased fracture rates (Loke et al. 2009, Solomon et al. 2009). Understanding the molecular mechanisms underlying TZD-induced side effects is central to the further development and successful therapeutic use of these drugs.

In rodent models, TZD-induced low bone mineral density and reduced bone formation have been attributed to the promotion of adipogenesis at the expense of osteogenesis (Johnson et al. 1999, Lecka-Czernik et al. 1999, Sottile \& Seuwen 2000, Shockley et al. 2007, Nishii et al. 2009, Cho et al. 2012). This is thought to be due to a shift in progenitor cell lineage allocation, as osteoblasts and adipocytes originate from the same

Published by Bioscientifica Ltd 
mesenchymal stem cell (MSC) population (Owen 1988). The commitment of MSCs during differentiation towards either adipogenesis or osteogenesis involves a stochastic mechanism under the control of lineage-specific transcription factors. Runt domain-containing protein, RUNX2 (also known as CBFA1 or OSF2), Osterix and msh homoeobox 2 (Msx2) play an essential role in osteoblast differentiation, and expression of these transcription factors is restricted to cells with an osteoblastic lineage (Ducy et al. 1997, Komori et al. 1997, Satokata et al. 2000, Nakashima et al. 2002, Nakashima \& de Crombrugghe et al. 2003). However, osteoblastic commitment may be radically usurped through activation of peroxisome proliferator-activated receptor gamma (Ppary (Pparg)), which promotes adipogenesis (Nishii et al. 2008). Nishii et al. (2008) demonstrated that treatment of immortalised bone marrow stromal cells (TBR31-2) with the Ppary agonist troglitazone resulted in a marked decrease in osteogenic-specific genes (Runx2, alkaline phosphatase $(A l p)$, bone Gla protein (Bgp (Bglap)), and type I collagen (Col1a1)) and induced the expression of the adipocytic markers adipsin (Adps) and lipoprotein lipase ( $L p l)$. Furthermore, Cho et al. (2012) indicated that rosiglitazone-induced activation of Ppar $\gamma$ reduced collagen and osteocalcin (BGLAP) levels in mouse primary bone marrowderived MSCs and prevented the mRNA expression of Runx2. These inhibitory effects of Ppary activation on osteoblastogenesis are further exemplified in Ppary null mutant embryonic stem cells, which spontaneously differentiate into osteoblasts, and in heterozygous Ppar $\gamma$ deficient mice, which exhibit high bone mass and increased osteoblastogenesis (Akune et al. 2004).

There are two distinct isoforms of Ppary (Zhu et al. 1995) in humans and mice. Ppary 1 (Pparg1) is predominantly expressed on osteoblasts, whereas Ppary2 (Pparg2) is specific to the adipocyte lineage (Lecka-Czernik et al. 1999, Ren et al. 2002). Naïve cells express lower levels of both Ppar 1 and Ppar 2 isoforms compared with lineage-committed cells (Sewter et al. 2002). Shockley et al. (2007) investigated the expression of gene markers associated with stem cell phenotype using DNA microarray analysis of U-33 stromal cells transfected with Ppar 2 (U-33/ $\gamma 2$ ) or vector control cells treated with TZDs. The authors demonstrated that rosiglitazone-induced activation of Ppary 2 down-regulates the expression of multiple stemness (Abcg2, Egfr, and Cd44) genes. Taken together, these results suggest that Ppar $\gamma$ negatively regulates stromal cell plasticity and promotes adipogenesis at the expense of osteogenesis.

These findings have recently been contradicted by Beck et al. (2013), who demonstrated that ex vivo TZD exposure stimulates adipogenesis, but does not directly alter osteoblast differentiation or lineage commitment in nondiabetic human bone marrow-derived MSCs. In the same study, the in vivo treatment of type 2 diabetic patients with rosiglitazone and pioglitazone did, however, decrease ex vivo osteogenic differentiation of bone marrow-derived MSCs, indicating an indirect negative effect on osteoblasts. This suggests that metabolic status influences the effects of TZDs on bone quality. The objective of this study was thus to investigate the influence of TZDs on both adipogenic and osteogenic differentiation of primary MSCs with distinct metabolic profiles. This study therefore utilised primary MSCs derived from either visceral (PV) or subcutaneous (SC) adipose tissue, as depot-specific metabolic differences exist between PV and SC adipose tissue (Dusserre et al. 2000, Lafontan \& Berlan 2003).

In addition to the metabolic influence as a possible explanation as to why studies utilising various Ppary agonists have yielded diverse results, another possible explanation for the diverse outcome of studies is that different types of TZDs are known to have distinct yet subtle difference in the affinity for Ppary activation. Generally, high-affinity Ppary ligands such as rosiglitazone, troglitazone, and pioglitazone decrease osteoblast proliferation and promote differentiation into an adipocytic phenotype (Johnson et al. 1999, Lecka-Czernik et al. 1999, Sottile \& Seuwen 2000), whereas low-affinity Ppar pan agonists, such as netoglitazone (Chang et al. 2007), have been reported to have a little negative effect on the skeleton in animal models. Lazarenko et al. (2006) investigated the pro-adipocytic and anti-osteoblastic activities of netoglitazone compared with those of rosiglitazone in immortalised U-33/ $\gamma 2$ stromal cells, primary bone marrow MSCs, and in vivo in C57BL/6 mice. The authors demonstrated that the expression of osteoblastspecific gene markers (Runx2, Dlx5, Bglap, and collagen) remained unchanged and that in vivo netoglitazone treatment did not result in trabecular bone loss.

As differences between various types of TZDs and metabolism can potentially influence the outcome of studies investigating the effect of TZD treatment on osteogenesis, this study aimed to directly compare the influence of rosiglitazone, pioglitazone (high affinity for Ppary), and netoglitazone (low affinity for Ppary) on the differentiation potential of primary MSCs with distinct metabolic profiles.

\section{Materials and methods}

This research study was approved by the Stellenbosch University Animal Ethics committee and complies with

Published by Bioscientifica Ltd 
the South African Animal Protection Act (Act no 71, 1962). All experiments were conducted in accordance with the South African Medical Research Council Guidelines on Ethics for Medical Research.

\section{Isolation of rat adipose-derived MSCs}

Male Wistar rats $(n=3)$ that were 10 weeks old with an average weight of $250 \pm 10$ (mean \pm s.D.) g were used in all experiments. Animals were killed by injecting $12 \mathrm{mg} / \mathrm{kg}$ sodium pentobarbitone (Eutha-naze Ref83/91, \#22079248DJ, Bayer), after which mesenchymal stromal cells were isolated from inguinal SC and peri-renal PV adipose tissues. The isolation procedure was adapted from Huang et al. (2002) as described previously (Sadie-Van Gijsen et al. 2010). Briefly, the excised tissue was rinsed with $75 \%(\mathrm{v} / \mathrm{v})$ ethanol (EtOH) and placed in high-glucose (4.5 g/l glucose; L-glutamine) DMEM (BioWhittaker, \#BE12-604F, Lonza, Basel, Switzerland). Tissue samples were diced and digested at $37^{\circ} \mathrm{C}$ for $30 \mathrm{~min}$ in $0.075 \%(\mathrm{w} / \mathrm{v})$ collagenase I (CLS1, \#XOM12195J, Worthington, Lakewood, NJ, USA) dissolved in Hanks' balanced salt solution (BioWhittaker, \#10-508F, Lonza) containing 1.5\% BSA. Digested samples were subjected to centrifugation for $5 \mathrm{~min}$ at $250 \boldsymbol{g}$ and the resulting pellets were washed with PBS. The pellets containing MSCs were re-suspended in DMEM containing 20\% foetal bovine serum (FBS; Biochrom, S0615, 0459B, Berlin, Germany) and seeded into two $100 \times 20 \mathrm{~mm}$ culture dishes (\#430167, Corning Life Sciences, Tewksbury, MA, USA). MSCs were maintained at $37^{\circ} \mathrm{C}$, in $90 \%$ humidified air with $5 \% \mathrm{CO}_{2}$. Growth media containing 20\% FBS were replaced after $24 \mathrm{~h}$ with standard growth media (SGM) containing high-glucose $(4.5 \mathrm{~g} / \mathrm{l})$ DMEM with $1 \%$ penicillin/streptomycin (BioWhittaker, \#DE17-6026, Lonza) supplemented with 10\% FBS (Biochrom, S0615, 0459B). For culture expansion, cells were treated with $0.5 \%$ trypsin $(200 \mathrm{mg} / \mathrm{l}$ Versene EDTA, \#BE171616, Lonza) and sub-cultured at a dilution of 1:4. All subsequent experimental procedures were carried out on cells in passage two and were repeated in triplicate wells for MSCs derived from three animals.

\section{MSC characterisation}

MSCs reaching $80 \%$ confluence in passage two under standard culturing conditions were harvested through trypsinisation and re-suspended in PBS containing 1\% BSA (\#A4503-100G, Sigma-Aldrich). Cell suspensions of $1 \times 10^{6}$ cells per $100 \mu \mathrm{l}$ were stained with mouse anti-rat FITC-conjugated CD90 (Thy-1; $5 \mu$ l, \#MR5001, Invitrogen) or FITC-conjugated CD45 (2.5 $\mu \mathrm{l}$, \#MR6901, Invitrogen) fluorescent antibodies. Flow cytometry was performed on the FACSCalibur using the CellQuest software. A total of 15000 events were recorded and data analysis was performed using the WinMDI 2.9 (J.Trotter) and Flow Jo Vx (Treestar) software.

\section{Medium conditions for differentiation}

MSCs that were 2 days post-confluence in passage two were used for all differentiation experiments. Standard adipogenic media (AM) contained SGM (refer to section 'Isolation of rat adipose-derived MSCs') supplemented with $10 \mu \mathrm{M}$ insulin (19278-5ML, SLBD5980, Sigma Life Sciences), $0.5 \mathrm{mM}$ 3-isobutyl-1-methylxanthine (IBMX, STBC7632V, Sigma Life Sciences), $1 \mu \mathrm{M}$ dexamethasone (D4902-100MG, BCBK1265V, Sigma Life Sciences), and $56 \mu$ M indomethacin (17378-5G, 064K1207, Sigma Life Sciences). Standard osteogenic differentiation media (OM) contained SGM supplemented with $10 \mathrm{nM}$ dexamethasone (D4902-100MG, BCBK1265V, Sigma Life Sciences), $50 \mu \mathrm{M}$ ascorbic acid (A4544-25G, SLBB4446V, Sigma Life Sciences), and $10 \mathrm{mM} \beta$-glycerophosphate (G5422-100G, 029K54241V, Sigma Life Sciences). Stock solutions of dexamethasone and indomethacin were prepared with ethanol, whereas IBMX and GW9662 (\#70785, Cayman Chemicals, Ann Arbor, MI, USA) stock solutions were prepared with dimethyl sulphoxide (DMSO, 1029873, Merck). Corresponding volumes of ethanol and DMSO were added to all cell culture conditions as vehicle controls.

\section{TZD treatment}

MSCs undergoing either adipogenesis or osteogenesis under differentiation conditions were treated with $1 \mu \mathrm{M}$ rosiglitazone (rosi), pioglitazone (pio), netoglitazone (neto) (Table 1) or indomethacin (indo; affinity for PPAR $\gamma: K_{\mathrm{d}} \sim 10^{-7} \mathrm{M}$, Lee et al. 2012). Stock solutions of TZDs were prepared with DMSO (1029873, Merck). A corresponding volume of DMSO was added to SGM for culturing untreated cells to serve as a vehicle control. MSCs were treated for 21 days during osteogenic differentiation with the media being changed twice weekly. For adipogenic differentiation, MSCs were treated for 14 days with the media being changed three times per week.

\section{Histology}

Lipid accumulation during both adipogenesis and osteogenic differentiation was assessed by Oil red $\mathrm{O}$ staining.

Published by Bioscientifica Ltd 
Table 1 Properties of thiazolidinediones used in this study

Rosiglitazone
Pioglitazone
Netoglitazone

\begin{tabular}{l} 
PPAR $\gamma$ affinity $\left(K_{\mathrm{d}}\right)$ \\
\hline $43 \mathrm{nM}^{\mathrm{b}}$ \\
$\mathrm{NA}$ \\
Ten- to 50 -fold lower \\
affinity than rosiglitazone \\
\hline
\end{tabular}

\begin{tabular}{c}
\hline PPAR $\gamma$ activation $\left(\mathrm{EC}_{50}, \mathrm{nM}\right)$ \\
\hline $30-200^{\mathrm{c}}$ \\
$400-500^{\mathrm{d}}$ \\
$8000^{\mathrm{e}}$ \\
\hline
\end{tabular}

Antihyperglycaemic potency ${ }^{\mathrm{a}}$ (ED25, mg/kg)

NA, not available.

${ }^{\mathrm{a}}$ Determined in $\mathrm{KK}-\mathrm{A}^{\mathrm{y}}$ mice.

${ }^{\mathrm{b}}$ Mouse PPAR $\gamma 1$ ligand-binding domain, Lehmann et al. (1995).

CLehmann et al. (1995), Reginato et al. (1998), and Sakamoto et al. (2000).

dLehmann et al. (1995) and Sakamoto et al. (2000).

${ }^{\mathrm{e}}$ Reginato et al. (1998).

Briefly, the media were discarded and cells were stained with $0.7 \%(\mathrm{w} / \mathrm{v})$ Oil red O prepared in $70 \%(\mathrm{v} / \mathrm{v})$ isopropanol. After $30 \mathrm{~min}$ at room temperature, the stain was removed and cells were washed three times with distilled water before being photographed for image analysis. Mineralisation during osteogenic differentiation was assessed by Alizarin red S staining. Briefly, cells were washed twice with PBS before being fixed in $70 \% \mathrm{EtOH}$ $(\mathrm{v} / \mathrm{v})$ for $5 \mathrm{~min}$. Fixed cells were washed with distilled water and stained with $100 \mathrm{mM}$ Alizarin red S (pH 4.0-4.1; 9436, Amresco, Solon, OH, USA) overnight at room temperature. Unbound Alizarin red $\mathrm{S}$ was removed by washing three times with distilled water and once with PBS before being photographed for image analysis. Image acquisition was carried out using an Olympus light microscope (CKX41, CachN $10 \times / 0.25$ PhP objective, EOS600D Canon digital camera). All experiments were carried out in triplicate for MSCs derived from three rats and four images (one image from each quartile) were taken per well. For each treatment condition, a total of 48 images were thus analysed using the ImageJ software (version 1.46, nih.gov). The extent of lipid accumulation or mineralisation was expressed as the percentage of positively stained surface area per well.

\section{RNA isolation and quantitative real-time PCR}

Total RNA was isolated on days 7 and 10 of differentiation using the RNeasy RNA isolation kit (Qiagen, \#74106) as per the manufacturer's instructions. DNase-treated RNA samples were used as templates for cDNA synthesis, using 20-(dT) as a primer with Promega ImProm-2 reverse transcriptase. Real-time semi-quantitative PCR was carried out on a Rotor-Gene (Qiagen) using the Quantace Sensimix kit. Relative gene expression (Table 2) was calculated according to the $\Delta C$ t method (Pfaffl 2001) by comparison

Table 2 Primer sequences and conditions used in semi-quantitative real-time PCR

\section{Transcripts}

(reference)

Ppary2 (Pparg2) (Tanabe et al. 2004)

Ap2 (Fabp4) (Fukuen et al. 2005)

$\operatorname{Adipsin}(A d p s)^{a}$

Msx2 (Kato et al. 2009)

Alkaline phosphatase (Alp) (Kuroda et al. 2005)

Collagen $1 \propto 1$ (An et al. 2006)

Arbp (Rp/p0) (van Wijngaarden et al. 2007)
Primer sequences $\left(5^{\prime}-3^{\prime}\right)$

F: ACT GCC TAT GAG CAC TTC AC

R: CAA TCG GAT GGT TCT TCG GA

F: TGA AAT CAC CCC AGA TGA CAG

R: CTC ATG CCC TTT CAT AAA CT

F: CAC GTG TGC GGT GGC ACC CTG

R: CCC CTG CAA GTG TCC CTG CGG T

F: TCA CCA CGT CCC AGC TTC TAG

R: AGC TTT TCC AGT TCC GCC TCC

F: GAG CAG GAA CAG AAG TTT GC

R: GTT GCA GGG TCT GGA GAG TA

F: GAC GTC CTG GTG AAG TTG G

R: AGC CAC GAT GAC CCT TTA TGR

F: AAA GGG TCC TGG CTT TGT CT

R: GCA AAT GCA GAT GGA TCG
Product size $(b p)$

448

194

476

178

202

588

91

aprimers designed based on the rat Adipsin gene sequence in GenBank: NCBI Reference Sequence NM_001077642.1.

http://joe.endocrinology-journals.org DOI: 10.1530/JOE-14-0425
(C) 2014 Society for Endocrinology Printed in Great Britain
Published by Bioscientifica Ltd. 
with the housekeeping gene acidic ribosomal phosphoprotein (Arbp (RplpO)) (van Wijngaarden et al. 2007).

\section{Statistical analysis}

Values are expressed as mean \pm s.D. Statistical analysis was performed using the Statistica software (StatSoft, version 10). Factorial ANOVA with Tukey's post hoc test was used to determine the group effect, treatment effect or group $\times$ treatment effect. Groups were classified as either SC MSCs or PV MSCs. Treatment effect was classified as the effect of a specific TZD on MSC differentiation in specific culture medium conditions. The level of significance was set at $P<0.05$.

\section{Results}

\section{MSC characterisation}

Primary MSCs isolated from SC and PV rat adipose tissue depots were characterised using cell surface markers to determine the purity of the expanded cell populations used for subsequent experiments. The progenitor cellassociated surface marker CD90 (Thy-1) was highly expressed on both SC $(99.4 \pm 0.3 \%)$ and PV $(97.3 \pm 1.1 \%)$ MSCs, whereas very few cells expressed the haematopoietic lineage-specific marker CD45 (SC 1.2 $\pm 1.0 \%$; PV 0.6 \pm $0.2 \%$ ) (Fig. 1). The isolated MSCs furthermore did not spontaneously differentiate and could thus be characterised as naïve.

\section{Adipogenesis}

No lipid accumulation was evident under SGM conditions (Fig. 2A and B). MSCs were cultured in standard adipogenic media (AM) in order to compare the rate of adipogenic differentiation between SC and PV MSCs. A higher percentage of lipid accumulation was evident in PV MSCs $(5.97 \pm 0.98 \%)$ compared with SC MSCs $(3.03 \pm 0.45 \%)(P<0.01$; Fig. $2 \mathrm{~A}$ and $\mathrm{B})$. The importance of Ppary activation during adipogenesis was assessed by either the addition of a Ppary antagonist (GW9662) or by the removal of the Ppary agonist (indomethacin) from the adipogenic media. Lipid accumulation was significantly $(P<0.001)$ reduced in both SC and PV MSCs in the presence of GW9662 and in the absence of indomethacin $\left(\mathrm{AM}^{\text {Indo--}}\right)$ (Fig. $2 \mathrm{~A}$ and B). The capacity of each of the TZDs to rescue adipogenesis in cells grown either in the presence of GW9662 or in the absence of indomethacin in adipogenic media (AM) was assessed. Rosiglitazone and
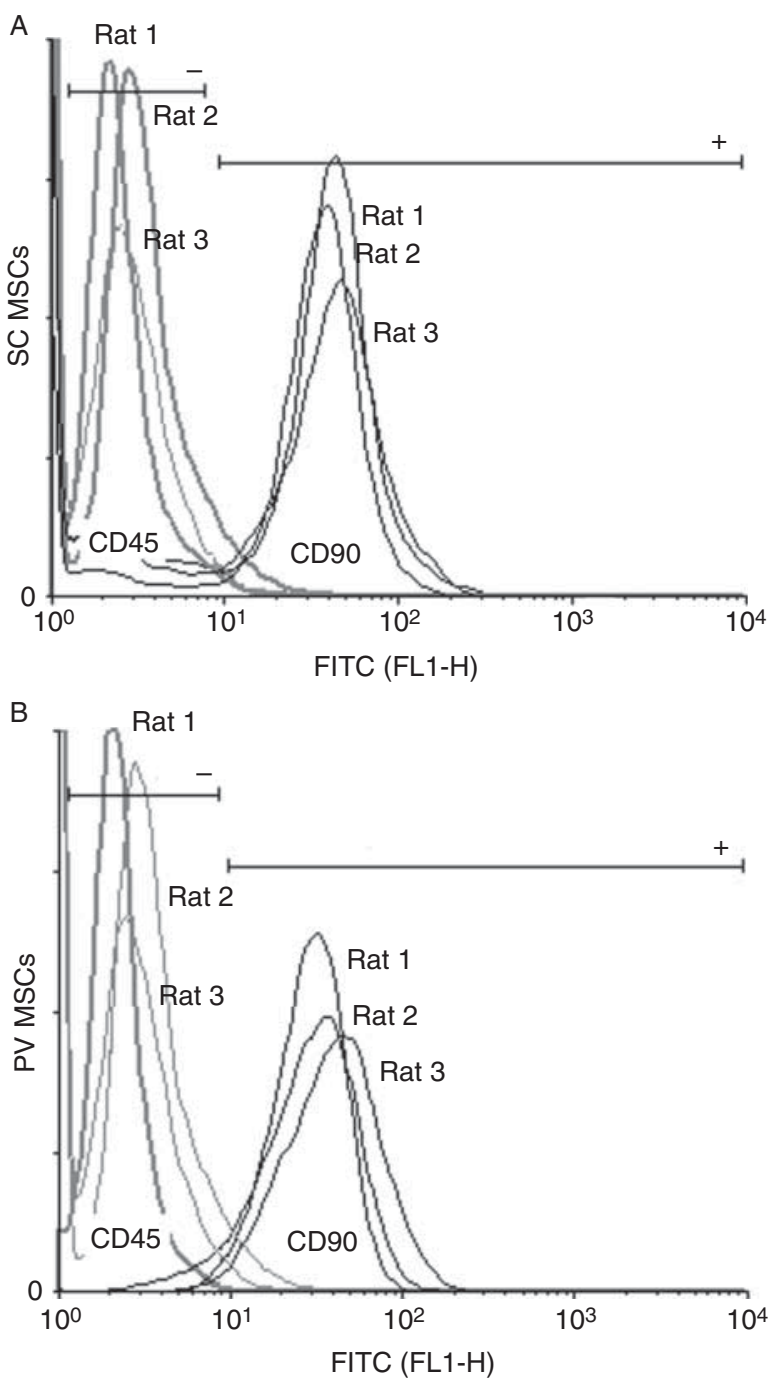

Figure 1

MSC surface marker expression. (A) SC MSCs: CD $90+C D 45-$. (B) PV MSCs: $\mathrm{CD} 90+\mathrm{CD} 45-$. A total of 15000 events were recorded, of which $9948 \pm$ 401 and $8977 \pm 745$ were gated as SC and PV respectively.

pioglitazone were able to partially rescue lipid accumulation (SC > PV MSCs) in the presence of GW9662 and in the absence of indomethacin from the differentiation media (Fig. 2C and D). Netoglitazone was unable to rescue lipid accumulation in the presence of GW9662 in either cell type, i.e. SC or PV MSCs (Fig. 2C). In the absence of indomethacin from the differentiation media, netoglitazone was, however, able to partially rescue lipid accumulation (PV > SC MSCs; Fig. 2D). Despite the lack of indomethacin and the addition of GW9662, rosiglitazone, and pioglitazone, but not netoglitazone, were still able to partially rescue adipogenesis (Fig. 2E). TZD treatment

Published by Bioscientifica Ltd 

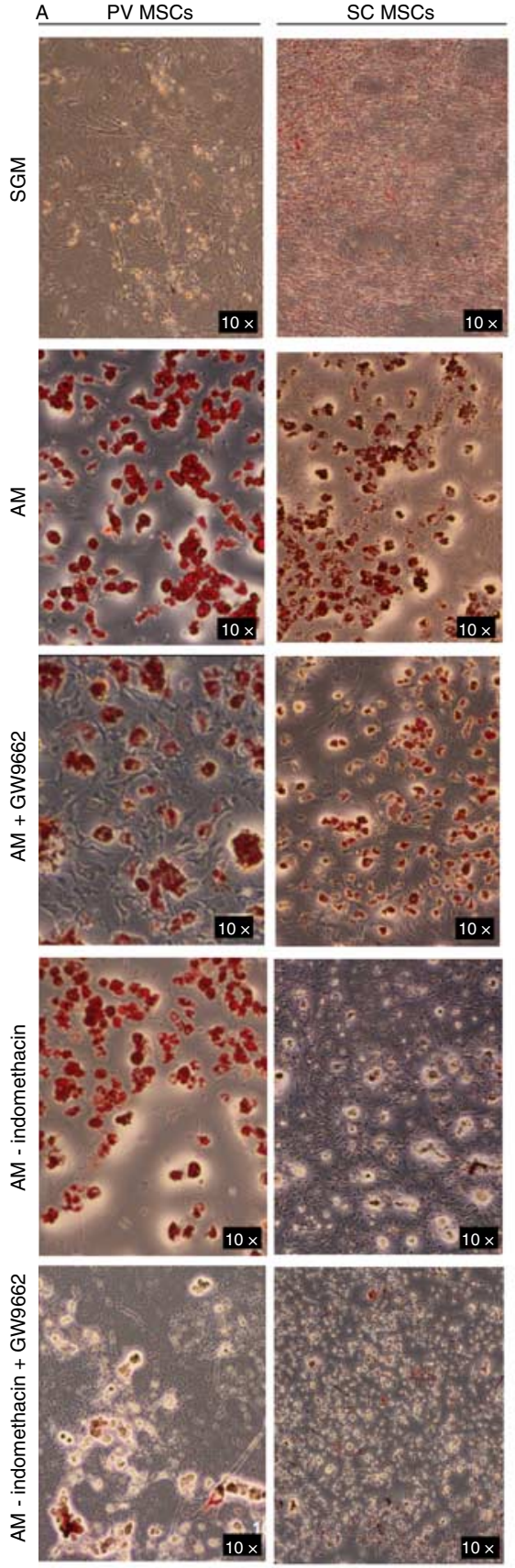

Adipogenesis: Oil Red O staining
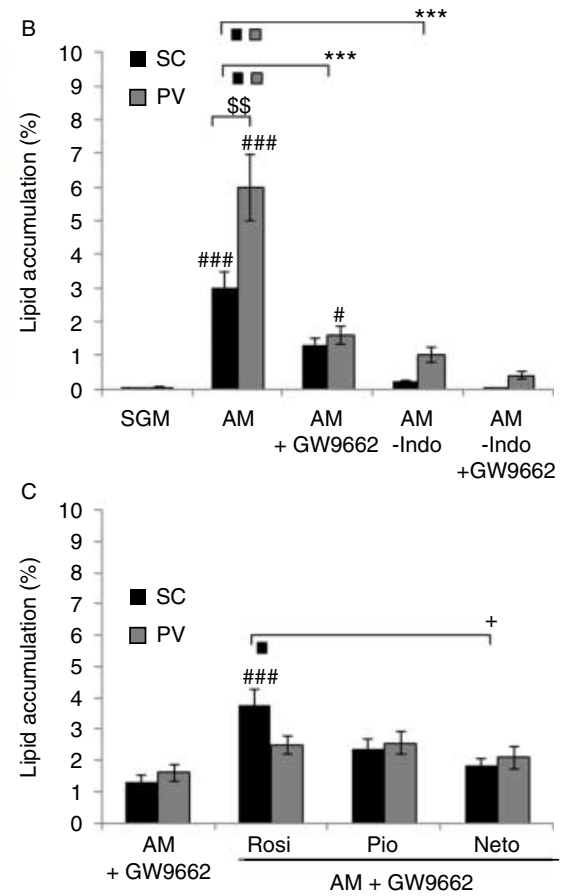

D
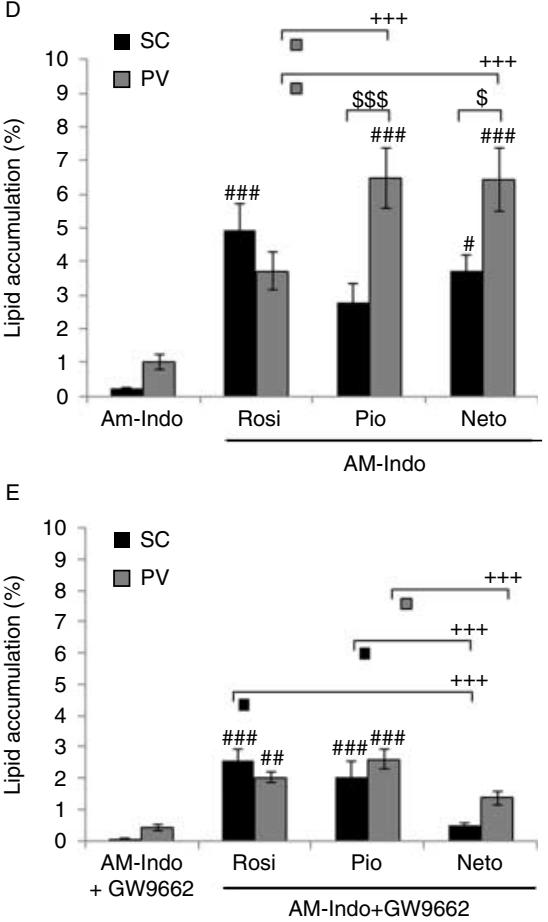

Factorial ANOVA with Tukey's post hoc test. ${ }^{\#} P<0.05$, ${ }^{\# \#} P<0.01$, and \#\#\# $P<0.001$ indicate significant difference from control media within the same cell type. ${ }^{+} P<0.05$ and ${ }^{+++} P<0.001$ indicate significant difference between TZD treatments in the same cell type. ${ }^{\$} P<0.05$ and ${ }^{\$ \$} P<0.001$ indicate significant difference between cell types within the same media and TZD treatment conditions. A full colour version of this figure available via http://dx.doi.org/10.1530/JOE-14-0425. http://joe.endocrinology-journals.org DOI: 10.1530/JOE-14-0425
(C) 2014 Society for Endocrinology Printed in Great Britain 
under standard adipogenic conditions (AM) did not enhance lipid accumulation compared with AM without TZD treatment (data not shown).

\section{Osteogenesis}

No mineralisation or lipid accumulation was evident in either SC or PV MSCs maintained in SGM (Fig. 3A and B). MSCs were cultured in standard OM in order to compare osteogenic differentiation between SC and PV MSCs. Mineralisation was evident in both SC $(10.3 \pm 2.0 \%)$ and PV MSCs $(8.6 \pm 1.1 \%)$ under standard OM conditions (Fig. 3B and C). The addition of the PPAR $\gamma$ antagonist GW9662, however, resulted in the highest levels of mineralisation (\% surface area stained positive for Alizarin red) in both cell types (SC MSCs $25.5 \pm 3.0 \%$ and PV MSCs $27.7 \pm 4.6 \%$ ) (Fig. 3B and C). To determine whether TZD treatment has a negative influence on osteogenesis, MSCs subjected to standard osteogenic conditions (OM) were treated with either rosiglitazone, pioglitazone or netoglitazone. TZD treatment did not negatively affect mineralisation in either SC or PV MSCs compared with OM without TZD treatment (Fig. 3C and E). In the presence of GW9662, TZD treatment did, however, reduce mineralisation compared with OM+GW9662 without TZD treatment $(P<0.01)$ (Fig. 3E), suggesting that TZD treatment counters the positive effect of GW9662 on mineralisation. Pronounced lipid accumulation was evident in PV MSCs during osteogenesis by treatment with indomethacin $(5.4 \pm 0.8 \%)$, rosiglitazone $(13.9 \pm 1.7 \%)$, or pioglitazone $(10.6 \pm 1.3 \%)$ compared with OM without TZD treatment $(0.4 \pm 0.1 \%)$ (Fig. $3 \mathrm{~F}$ and $\mathrm{G})$. However, netoglitazone treatment did not result in lipid accumulation during osteogenesis (Fig. 3F). During osteogenesis, PV MSCs were
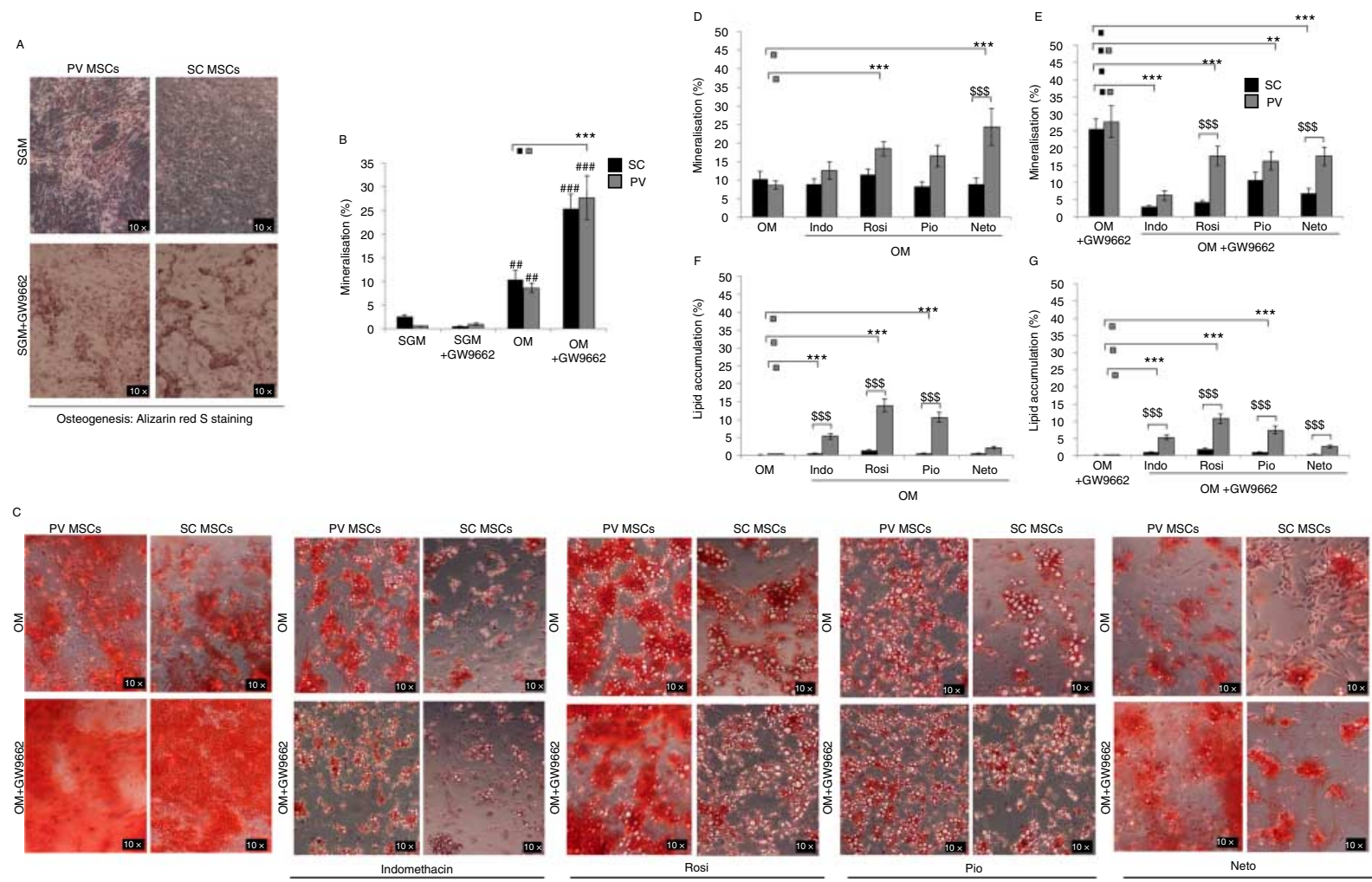

Figure 3

Osteogenesis: mineralisation. (A) No evidence of mineralisation in SGM with or without PPAR $\gamma$ antagonist (GW9662). (B) Mineralisation in different medium conditions without TZD treatment. (C) Alizarin red S staining (day 21) demonstrating mineralisation with lipid droplets evident with TZD treatment. (D) Mineralisation with TZD treatment in OM. (E) Mineralisation with TZD treatment in OM + GW9662. (F) Lipid accumulation with TZD treatment in OM. (G) Lipid accumulation with TZD treatment in OM + GW9662. Statistical analysis: factorial ANOVA with
Tukey's post hoc test. ${ }^{\# \#} P<0.01$ and ${ }^{\# \#} P<0.001$ indicate significant difference between SGM and other medium conditions (B). ${ }^{*} P<0.01$ and $* * * P<0.001$ indicate significant difference between $\mathrm{OM}$ and $\mathrm{OM}+$ GW9662 (B) and significant TZD treatment effect (D, E, F and G) in the same cell type. ${ }^{\$ \$} P<0.001$ indicate significant difference between $S C$ and $P V$ MSCs in the same treatment conditions. A full colour version of this figure available via http://dx.doi.org/10.1530/JOE-14-0425.

Published by Bioscientifica Ltd. 
more prone to lipid accumulation than SC MSCs in response to TZD treatment (Fig. $3 \mathrm{~F}$ and G). Lipid droplet accumulation was furthermore evident in areas of mineralisation (Fig. 3C) in both SC and PV MSCs.

\section{Relative mRNA expression of adipogenic genes}

The relative gene expression levels of the adipocytic genes Ppary2, Ap2 (Fabp4), and Adipsin were determined to assess whether TZD treatment skews osteogenesis towards adipogenesis. Consistent with histological observations, PV MSCs were more adipogenic than SC MSCs. In PV MSCs, on day 10 of adipogenic differentiation (AM), the expression of adipogenic genes (Ppary2, Ap2, and Adipsin) was significantly elevated $(P<0.01)$ compared with control conditions (SGM). Despite significant lipid accumulation observed with TZD treatment during osteogenesis, Ppary 2 expression during rosiglitazone, pioglitazone, and netoglitazone treatment was less compared with AM and not significantly different from control media (SGM; Fig. 4A and B). Rosiglitazone and pioglitazone treatment induced modest expression of Ap2 (relative expression, $\mathrm{AM}=1$; Rosi $0.53 \pm 0.2$; Pio $0.47 \pm 0.2$ ) on day 10 in PV MSCs, whereas netoglitazone did not (relative expression, $\mathrm{AM}=1 ; 0.03 \pm 0.01$ ) (Fig. $4 \mathrm{C}$ and D). TZD treatment during osteogenesis was, however, unable to induce the expression of adipsin (Fig. 4E and F).

\section{Relative mRNA expression of osteogenic genes}

The relative gene expression levels of the osteogenic genes, Msx2, Collagen I, and Alp, were assessed to determine whether TZD treatment has a negative influence on osteogenesis. TZD treatment did not downregulate the expression of the pro-osteogenic genes (Ms 2 2, Collagen I, and $A l p$ ) during osteogenesis (Fig. 5A, B, C, D, E, and F). In PV MSCs, pioglitazone treatment increased Msx2 expression after 10 days of osteogenic differentiation, whereas no effect was observed in SC MSCs (Fig. 5A and B).

\section{Discussion}

This is the first investigation to directly compare the effect of three different TZDs on the differentiation of MSCs towards the adipogenic or osteogenic lineage. Uniquely, it was found that although TZD treatment did not negatively affect mineralisation, Ppary ligands (indomethacin, rosiglitazone, and pioglitazone) promoted the accumulation of lipid droplets during osteogenesis. In addition, the partial inhibition of basal Ppary activity under standard osteogenic conditions markedly increased mineralisation. The absence of a Ppary agonist from the adipogenic media or the addition of a Ppary antagonist reduced lipid accumulation and indicated the known importance of Ppary activation during adipogenesis (Lecka-Czernik et al. 2002). The direct treatment comparison between three different TZDs demonstrated that unlike rosiglitazone and pioglitazone, the Ppar pan agonist, netoglitazone, was not able to rescue adipogenesis in the presence of the Ppary antagonist GW9662 and induced the least lipid accumulation during osteogenesis. Despite indirect evidence pointing towards the skewing of MSC differentiation towards adipogenesis with TZD treatment, the expression of the adipogenic genes Ppary and Ap2 was only modestly increased compared with SGM, whereas the expression of the adipocyte-specific marker Adipsin was not induced by TZD treatment. Taken together, the data therefore suggest that aberrant lipid droplet formation occurred concurrently with mineralisation and that differentiation was not overtly skewed towards adipogenesis.

The negative influence of TZDs on bone mineral density has been as attributed to the promotion of adipogenesis at the expense of osteogenesis, i.e. reduced bone formation due to a shift in MSC lineage allocation. Challenging this generally accepted view, Bruedigam et al. (2010) proposed an alternative concept explaining the detrimental effects of TZDs on bone. The authors utilised human preosteoblasts and osteoblasts that were already committed to the osteogenic lineage and demonstrated that the addition of rosiglitazone to osteogenic differentiation medium accelerated osteoblast differentiation and was followed by an accumulation of reactive oxygen species that lead to osteoblast apoptosis, whereas cells from the adipogenic lineage (adipocytes) were not apoptotic following rosiglitazone treatment. In the same investigation, MSCs pre-treated with rosiglitazone did not preferentially differentiate into adipocytes. Taken together, these findings suggest that cells of the osteogenic lineage are more susceptible to TZD-induced apoptosis than cells of the adipogenic lineage (Bruedigam et al. 2010). The authors concluded that compared with adipocytes, oxidative stress and apoptosis are preferentially triggered in osteoblasts by activated Ppary, explaining the suppressive action of TZD treatment on bone (Bruedigam et al. 2010). In the current investigation, TZD treatment during osteogenesis (without GW9662) did not negatively influence mineralisation. The addition of a Ppary antagonist (GW9662) to

Published by Bioscientifica Ltd. 

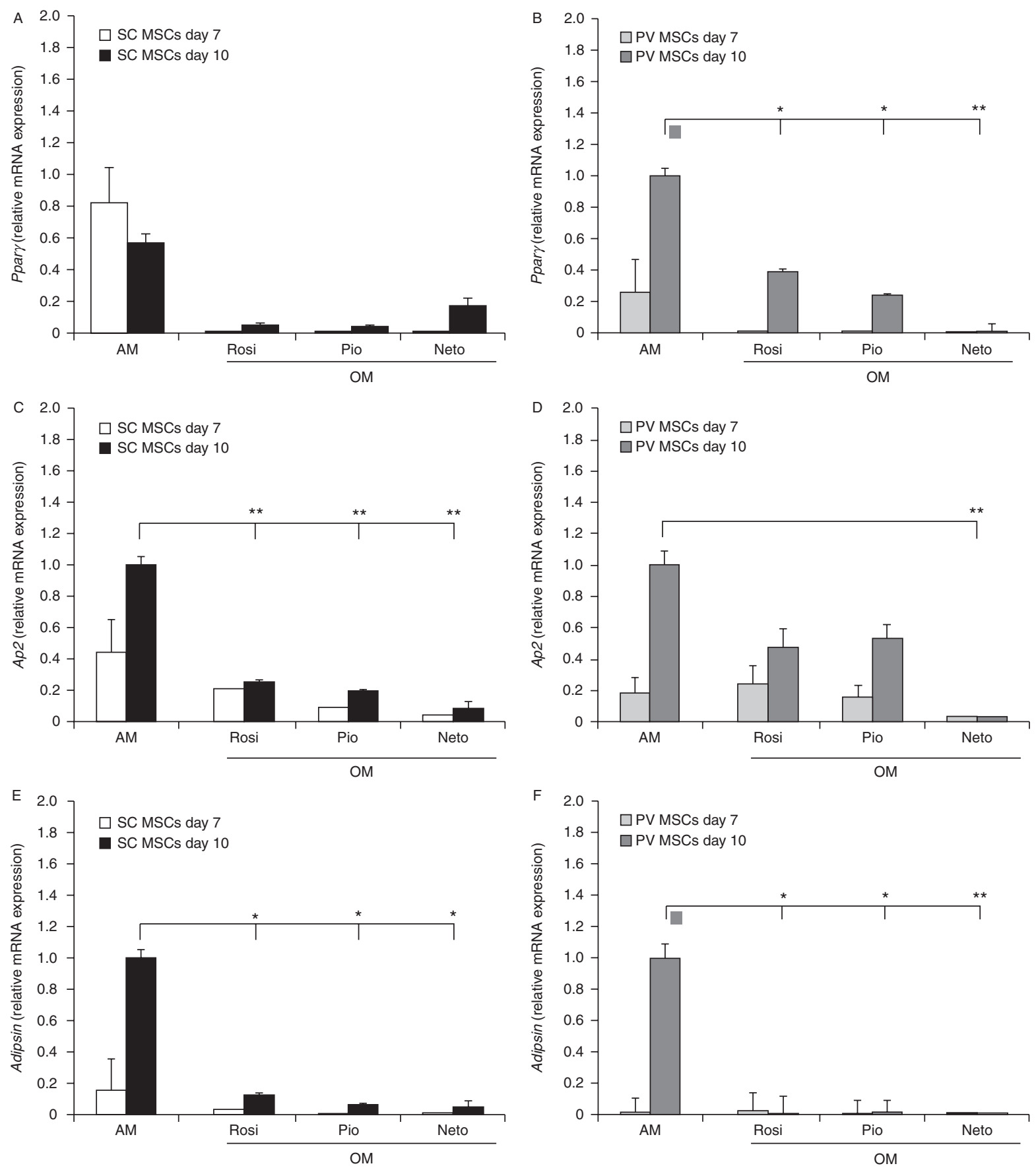

Figure 4

Pro-adipogenic gene expression. The relative mRNA expression of Ppar 2 (A and B), Ap2 (C and D), and Adipsin (E and F) in SC MSCs and PV MSCs. Statistical analysis: factorial ANOVA with Tukey's post hoc test. ${ }^{*} P<0.05$

osteogenic media without TZD treatment did, however, significantly increased mineralisation. In an investigation similar to that by Bruedigam et al. (2010), Wang et al. (2012) investigated the effect of TZD treatment on

and $* * P<0.01$ indicate significant treatment effect compared with AM at the same time point. Note: values are expressed as the relative expression of Ppary2, Ap2, or Adipsin compared with the housekeeping gene, Arbp.

the trans-differentiation of preosteoblasts (bone marrowderived MSCs differentiated towards the osteogenic lineage) into adipocytes. Following either 5 or 14 days of osteogenic differentiation of MSCs, osteogenic media

Published by Bioscientifica Ltd. 

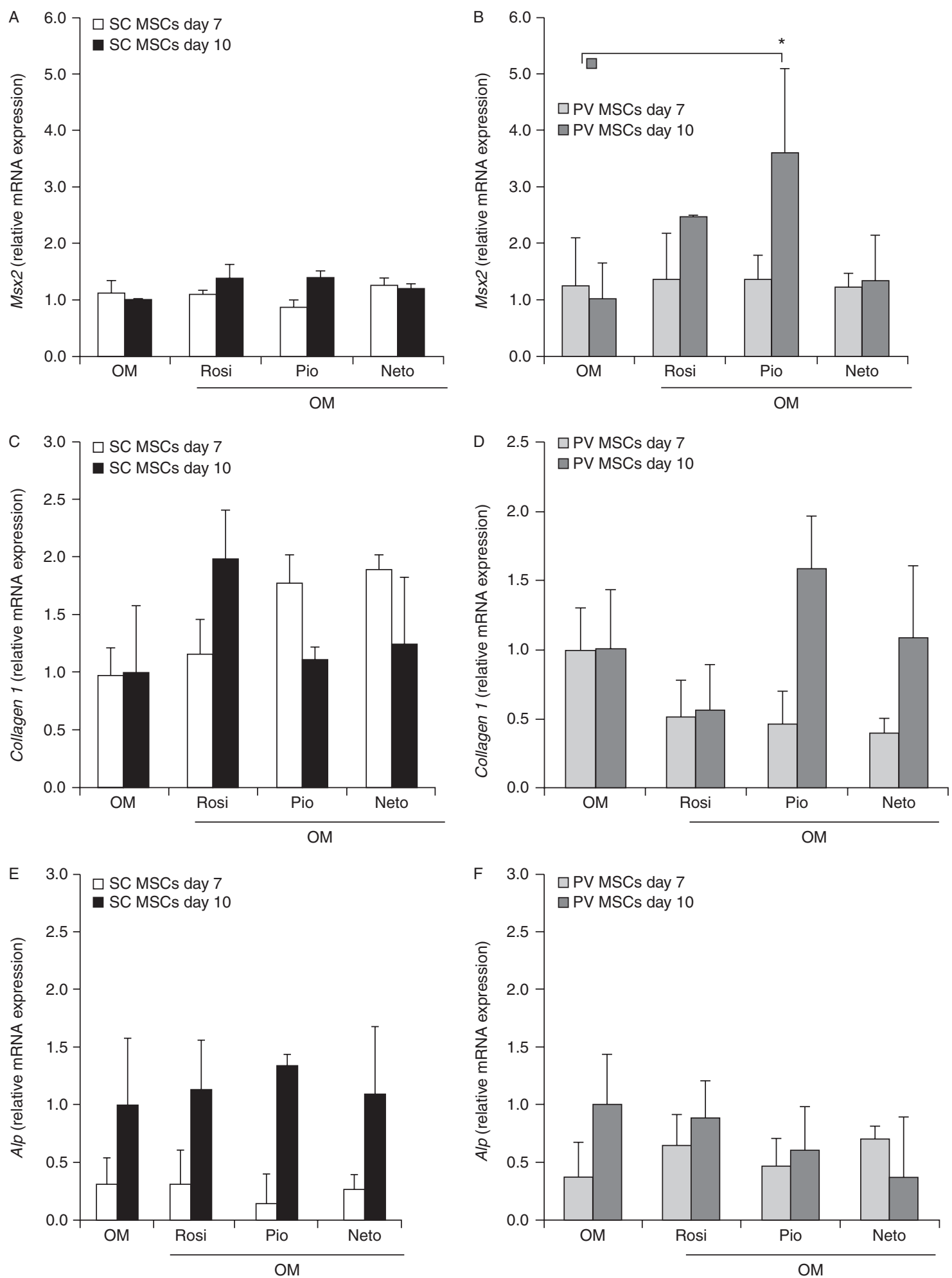

Figure 5

Pro-osteogenic gene expression. The relative mRNA expression of $M s \times 2$ ( $A$ and B), Collagen I ( $C$ and D), and alkaline phosphatase ( $E$ and F) in SC MSCs and PV MSCs. Statistical analysis: factorial ANOVA with Tukey's post hoc test.

${ }^{*} P<0.05$ indicate significant treatment effect compared with $\mathrm{OM}$ at the same time point. Note: values are expressed as the relative expression of Msx2, Collagen I, or Alp compared with the housekeeping gene, Arbp. 
were changed to adipogenic media with or without the addition of pioglitazone (21 days). The authors demonstrated that MSCs subjected to osteogenic conditions for shorter durations retained a stronger ability to differentiate into adipocytes. Trans-differentiation was furthermore enhanced by pioglitazone treatment and was associated with decreased mRNA expression of osteogenic genes (Runx2 and Alp) and increased Ppary expression.

In the current investigation, simultaneous mineralisation and lipid droplet formation were evident with indomethacin, rosiglitazone, and pioglitazone treatment during osteogenesis. Despite modest increases in the expression of $A p 2$ with rosi- and pio-treatment, the change in Ppary levels was insufficient to induce the downstream expression of the adipocyte-specific marker, Adipsin (Duan et al. 2014). Novel data from this study therefore suggest that lipid droplet formation during mineralising conditions may not necessarily be the result of the skewing of differentiation towards adipogenesis. Netoglitazone treatment during osteogenesis did, however, result in less lipid accumulation compared with rosiglitazone and pioglitazone treatment, TZDs that have a high affinity for Ppary, which may be due to the limited ability of netoglitazone to activate Ppary. This is consistent with in vitro studies utilising U-33 stromal cells transfected with Ppary2 (U-33/y2), vector control cells (U-33/c) and primary bone marrow MSC cultures, which showed that netoglitazone is less effective than rosiglitazone in inducing lipid accumulation (Lazarenko et al. 2006). Contradicting the in vitro observations, analysis of the trabecular bone marrow composition in the proximal tibia of CD7BC/6 mice following 7 weeks of TZD treatment demonstrated similar increases in adiposity with netoglitazone and rosiglitazone treatment (Lazarenko et al. 2006). The authors did, however, demonstrate that despite the accumulation of adipocytes, chronic netoglitazone administration had little to no negative effect on the skeleton in vivo (Lazarenko et al. 2006). Taken together with the above findings, data from the current investigation suggest that the extent of TZD-induced lipid accumulation is highly dependent on the nature of these Ppar ligands.

Lecka-Czernik et al. (2002) have previously investigated the influence of diverse Ppary ligands on MSC (Swiss-Webster mice bone marrow-derived MSCs and $\mathrm{U}-33 / \gamma 2$ ) differentiation. The authors demonstrated that, although some naturally occurring Ppary ligands (9,10-dihydroxyoctadecanoic acid and 15-deoxy- $\Delta^{12,14}$ $\mathrm{PGJ}_{2}$ ) stimulate adipogenesis and inhibit osteoblast differentiation, other ligands (9,10-epoxyoctadecanoic acid and thiazolidine acetamide ligand GW0072) prevent osteogenesis but do not stimulate adipogenesis. The authors suggested that the pro-adipogenic and antiosteoblastogenic effects of Ppary are regulated by diverse downstream regulatory pathways that can be differentially modulated depending on the nature of the ligand. Ppar pan-agonist and dual-agonist drugs such as netoglitazone were thus developed to combine the triglyceride-lowering and HDL cholesterol-elevating effects of the Ppar (Ppara) agonists with the insulin sensitisation elicited by the Ppar agonists (Liu et al. 1998, Upton et al. 1998, Pickavance et al. 1998, 2003, Chang et al. 2007), while limiting negative effects on the skeleton (Lazarenko et al. 2006). Unfortunately, to date, Ppary/Ppar $\alpha$ dual agonist treatment is associated with unacceptable adverse effects that have delayed clinical application of these drugs (Chang et al. 2007). In addition to the diverse nature of Ppar ligands, the current study also demonstrated that the metabolic status of MSCs influences the ability of TZDs to induce lipid droplet accumulation in both adipogenic and osteogenic medium conditions.

SC adipose tissue has a higher propensity for lipogenesis and fatty acid uptake than its PV counterpart (for review, see Lafontan et al. (2003)). Nonetheless, this study demonstrated that, compared with SC MSCs, PV MSCs are more prone to lipid accumulation under controlled differentiation conditions in vitro. The difference in the extent of lipid accumulation between PV and SC MSCs can in part be explained by their divergent lipogenic responses to insulin. Sadie-Van Gijsen et al. (2010) demonstrated that insulin contributes to lipid accumulation in PV MSCs but is not essential for adipogenesis in SC MSCs. The authors furthermore demonstrated that the presence of insulin in adipogenic media increased SC MSC proliferation and suggested that, in hyper-insulinaemic states, SC MSCs increase in number while maintaining intracellular lipid content in a non-pathological range, whereas PV MSCs are more prone to hypertrophy during lipid accumulation. In this study, the substitution of indomethacin with various TZDs in adipogenic differentiation media demonstrated that MSCs derived from adipose tissue with diverse lipogenic responses to insulin also have a diverse response to specific TZDs. PV MSCs were more responsive to pioglitazone and netoglitazone, whereas SC MSCs had a higher percentage of lipid accumulation when treated with rosiglitazone. Furthermore, during osteogenesis, the addition of indomethacin, rosiglitazone, and pioglitazone induced lipid droplet formation only in PV MSCs. This study is thus the first to demonstrate that metabolically

Published by Bioscientifica Ltd. 
diverse mesenchymal stromal cells respond differently to in vitro TZD treatment.

\section{Conclusion}

It is important to identify whether factors such as obesity and altered metabolism in addition to drug treatment can affect stem cell behaviour specifically because of the fact that, in the context of type 2 diabetes, patients often have altered metabolic profiles. Owing to the severity of the 'off-target' effects of TZDs, clinical administration of these drugs has been under the spotlight in recent years (Yau et al. 2013). Pioglitazone is, however, still available for clinical use despite the potential long-term adverse effects, such as 'fatty bones', which may progress towards osteoporosis. The novel evidence in this study demonstrates that lipid droplet formation and mineralisation can occur concurrently in response to TZD treatment during osteogenesis and that lipid accumulation was more pronounced in MSCs derived from peri-renal PV adipose tissue than in MSCs derived from its SC counterpart. This investigation furthermore demonstrates that the extent of lipid accumulation is dependent on the nature of the Ppar ligand and that metabolic status can contribute to the adverse effects associated with TZD treatment. Unfortunately, owing to the limitations associated with the in vitro nature of this study, the observations cannot be extrapolated to the in vivo situation. Future studies will therefore focus on in vivo TZD treatment models, comparing the ex vivo differentiation of bone marrowderived MSCs from healthy/normal and obese animals.

\section{Declaration of interest}

The authors declare that there is no conflict of interest that could be perceived as prejudicing the impartiality of the research reported.

\section{Funding}

This work was supported by research funding from the South African Medical Research Council and the South African National Research Foundation. Dr E A was supported by a postdoctoral fellowship from the National Research Foundation. Dr M v and Dr I L C were supported by postdoctoral fellowships from the Faculty of Medicine and Health Sciences, Stellenbosch University.

\section{Author contribution statement}

All the authors contributed to the conceptual design of the study and interpretation of data. Drs M V, E A, and I L C were involved in the experimental procedures, sample collection and sample analysis. $\mathrm{Dr} M \mathrm{v}$ was responsible for data analysis and writing of the manuscript. Prof. W F F contributed to the interpretation of data and editing of the manuscript.

\section{References}

Akune T, Ohba S, Kamekura S, Yamaguchi M, Chung U-I, Kubota N, Terauchi Y, Harada Y, Azuma Y, Nakamura K et al. 2004 PPAR $\gamma$ insufficiency enhances osteogenesis through osteoblast formation from bone marrow progenitors. Journal of Clinical Investigation 113 846-855. (doi:10.1172/JCI200419900)

An SJ, Boyd R, Wang Y, Qiu X \& Wang HD 2006 Endothelin-1 expression in vascular adventitial fibroblasts. American Journal of Physiology. Heart and Circulatory Physiology 290 H700-H708. (doi:10.1152/ ajpheart.00326.2005)

Beck GR Jr, Khazai NB, Bouloux GF, Camalier CE, Lin Y, Garneys LM, Siqueira J, Peng L, Pasquel F, Umpierrez D et al. 2013 The effects of thiazolidinediones on human bone marrow stromal cell differentiation in vitro and in thiazolidinedione-treated patients with type 2 diabetes. Translational Research 161 145-155. (doi:10.1016/j.trsl.2012.08.006)

Bergeron R, Yao J, Woods JW, Zycband EI, Liu C, Li Z, Adams A, Berger JP, Zhang BB, Moller DE et al. 2006 Peroxisome proliferator-activated receptor (PPAR)- $\alpha$ agonism prevents the onset of type 2 diabetes in Zucker diabetic fatty rats: a comparison with PPAR $\gamma$ agonism. Endocrinology 147 4252-4262. (doi:10.1210/en.2005-1535)

Bruedigam C, Eijken M, Koedam M, van de Peppel J, Drabek K, Chiba H \& van Leeuwen JP 2010 A new concept underlying stem cell lineage skewing that explains the detrimental effects of thiazolidinediones on bone. Stem Cells 28 916-927. (doi:10.1002/stem.405)

Cariou B, Charbonnel B \& Staels B 2012 Thiazolidinediones and PPAR $\gamma$ agonists: time for a reassessment. Trends in Endocrinology and Metabolism 23 205-215. (doi:10.1016/j.tem.2012.03.001)

Chang F, Jaber LA, Berlie HD \& O'Connell MB 2007 Evolution of peroxisome proliferator-activated receptor agonists. Annals of Pharmacotherapy 41 973-983. (doi:10.1345/aph.1K013)

Cho E-S, Kim M-K, Son Y-O, Lee K-S, Park S-M \& Lee J-C 2012 The effects of rosiglitazone on osteoblastic differentiation, osteoclast formation and bone resorption. Molecules and Cells 33 173-181. (doi:10.1007/ s10059-012-2240-z)

Duan Z, Zhao X, Fu X, Su C, Xin L, Saarikettu J, Yang X, Yao Z, Silvennoinen O, Wei M etal. 2014 Tudor-SN, a novel coactivator of peroxisome proliferatoractivated receptor $\gamma$ protein, is essential for adipogenesis. Journal of Biological Chemistry 289 8364-8374. (doi:10.1074/jbc.M113.523456)

Ducy P, Zhang R, Geoffroy V, Ridall AL \& Karsenty G 1997 Osf2/Cbfa1: a transcriptional activator of osteoblast differentiation. Cell 89 747-754. (doi:10.1016/S0092-8674(00)80257-3)

Dusserre E, Moulin P \& Vidal H 2000 Differences in mRNA expression of the proteins secreted by the adipocytes in human subcutaneous and visceral adipose tissues. Biochimica et Biophysica Acta 1500 88-96. (doi:10.1016/S0925-4439(99)00091-5)

Fukuen S, Iwaki M, Yasui A, Makishima M, Matsuda M \& Shimomura I 2005 Sulfonylurea agents exhibit peroxisome proliferator-activated receptor $\gamma$ agonistic activity. Journal of Biological Chemistry $28023653-23659$. (doi:10.1074/jbc.M412113200)

Gitt AK, Bramlage P, Binz C, Krekler M, Deeg E \& Tschöpe D 2012 Comorbidity, hypoglycaemia and appropriate selection of antidiabetic pharmacotherapy in diabetic patients with heart failure in clinical practice in Germany. Results of the DiaRegis registry. Herz 37 294-300. (doi:10.1007/s00059-012-3611-3)

Huang JI, Beanes SR, Zhu M, Lorenz HP, Hedrick MH \& Benhaim P 2002 Rat extramedullary adipose tissue as a source of osteochondrogenic progenitor cells. Plastic and Reconstructive Surgery 109 1033-1043.

Johnson TE, Vogel R, Rutledge SJ, Rodan G \& Schmidt A 1999 Thiazolidinedione effects on glucocorticoid receptor-mediated gene transcription and differentiation in osteoblastic cells. Endocrinology 140 3245-3254. (doi:10.1210/endo.140.7.6797)

Karalliedde J, Buckingham R, Starkie M, Lorand D, Stewart M, Viberti G \& Rosiglitazone Fluid Retention Study Group 2006 Effect of various diuretic treatments on rosiglitazone-induced fluid retention. http://joe.endocrinology-journals.org DOI: $10.1530 /$ JOE-14-0425
() 2014 Society for Endocrinology Printed in Great Britain 
Journal of the American Society of Nephrology 17 3482-3490. (doi:10.1681/ASN.2006060606)

Kato S, Kawabata N, Suzuki N, Ohmura M \& Takagi M 2009 Bone morphogenetic protein-2 induces the differentiation of a mesenchymal progenitor cell line, ROB-C26, into mature osteoblasts and adipocytes. Life Sciences 84 302-310. (doi:10.1016/j.lfs.2008.12.011)

Komori T, Yagi H, Nomura S, Yamaguchi A, Sasaki K, Deguchi K, Shimizu Y, Bronson RT, Gao YH, Inada M et al. 1997 Targeted disruption of Cbfa1 results in a complete lack of bone formation owing to maturational arrest of osteoblasts. Cell 89 755-764. (doi:10.1016/S00928674(00)80258-5)

Kuroda S, Virdi AS, Dai Y, Shott S \& Sumner DR 2005 Patterns and localization of gene expression during intramembranous bone regeneration in the rat femoral marrow ablation model. Calcified Tissue International 77 212-225. (doi:10.1007/s00223-004-0267-x)

Lafontan M \& Berlan M 2003 Do regional differences in adipocyte biology provide new pathophysiological insights? Trends in Pharmacological Sciences 24 276-283. (doi:10.1016/S0165-6147(03)00132-9)

Lazarenko OP, Rzonca SO, Suva LJ \& Lecka-Czernik B 2006 Netoglitazone is a PPAR- $\gamma$ ligand with selective effects on bone and fat. Bone 38 74-84. (doi:10.1016/j.bone.2005.07.008)

Lecka-Czernik B, Gubrij I, Moerman EJ, Kajkenova O, Lipschitz DA, Manolagas SC \& Jilka RL 1999 Inhibition of Osf2/Cbfa1 expression and terminal osteoblast differentiation by PPAR $\gamma 2$. Journal of Cellular Biochemistry 74 357-371. (doi:10.1002/(SICI)1097-4644 (19990901)74:3<357::AID-JCB5> 3.0.CO;2-7)

Lecka-Czernik B, Moerman EJ, Grant DF, Lehmann JM, Manolagas SC \& Jilka RL 2002 Divergent effects of selective peroxisome proliferatoractivated receptor- $\gamma 2$ ligands on adipocyte vs osteoblast differentiation. Endocrinology 143 2376-2384. (doi:10.1210/endo.143.6.8834)

Lee J-Y, Kim J-K, Lee S-J, Lee E-J, Shin S-Y, Jin Q, Yoon D-Y, Woo E-R \& Kim Y-M 2012 Binding model of amentoflavone to peroxisome proliferator-activated receptor $\gamma$. Bulletin of the Korean Chemical Society 33 1475-1479. (doi:10.5012/bkcs.2012.33.5.1475)

Lehmann JM, Moore LB, Smith-Oliver TA, Wilkison WO, Willson TM \& Kliewer SA 1995 An antidiabetic thiazolidinedione is a high affinity ligand for peroxisome proliferator-activated receptor $\gamma(\operatorname{PPAR} \gamma)$. Journal of Biological Chemistry 270 12953-12956. (doi:10.1074/jbc.270. 22.12953)

Liu LS, Tanaka H, Ishii S \& Eckel J 1998 The new antidiabetic drug MCC-555 acutely sensitizes insulin signaling in isolated cardiomyocytes. Endocrinology 139 4531-4539. (doi:10.1210/endo.139.11.6310)

Loke YK, Singh S \& Furberg CD 2009 Long-term use of thiazolidinediones and fractures in type 2 diabetes: a meta-analysis. Canadian Medical Association Journal 180 32-39. (doi:10.1503/cmaj.080486)

Mamtani R, Haynes K, Bilker WB, Vaughn DJ, Strom BL, Glanz K \& Lewis JD 2012 Association between longer therapy with thiazolidinediones and risk of bladder cancer: a cohort study. Journal of the National Cancer Institute 104 1411-1421. (doi:10.1093/jnci/djs328)

Nakashima K \& de Crombrugghe B 2003 Transcriptional mechanisms in osteoblast differentiation and bone formation. Trends in Genetics 19 458-466. (doi:10.1016/S0168-9525(03)00176-8)

Nakashima K, Zhou X, Kunkel G, Zhang Z, Deng JM, Behringer RR \& de Crombrugghe B 2002 The novel zinc finger-containing transcription factor osterix is required for osteoblast differentiation and bone formation. Cell 108 17-29. (doi:10.1016/S0092-8674(01) 00622-5)

Nishii N, Arai M, Yanai N, Togari A \& Nakabayashi T 2009 Effect of bone morphogenetic protein-2 (BMP-2) or troglitazone, as an inducer of osteogenic cells or adipocytes, on differentiation of a bone marrow mesenchymal progenitor cell line established from temperaturesensitive (ts) simian virus (SV) 40 T-antigen gene transgenic mice. Biological and Pharmaceutical Bulletin 32 10-17. (doi:10.1248/bpb.32.10)

Owen M 1988 Marrow stromal stem cells. Journal of Cell Science. Supplement 10 63-76. (doi:10.1242/jcs.1988.Supplement_10.5)
Pfaffl MW 2001 A new mathematical model for relative quantification in real-time RT-PCR. Nucleic Acids Research 29 e45. (doi:10.1093/ nar/29.9.e45)

Pickavance L, Widdowson PS, King P, Ishii S, Tanaka H \& Williams G 1998 The development of overt diabetes in young Zucker diabetic fatty (ZDF) rats and the effects of chronic MCC-555 treatment. British Journal of Pharmacology 125 767-770. (doi:10.1038/sj.bjp.0702158)

Pickavance LC, Widdowson PS, Foster JR, Williams G \& Wilding JPH 2003 Chronic treatment with the thiazolidinedione, MCC-555, is associated with reductions in nitric oxide synthase activity and $\beta$-cell apoptosis in the pancreas of the Zucker diabetic fatty rat. International Journal of Experimental Pathology 84 83-89. (doi:10.1046/j.1365-2613.2003.00337.x)

Reginato MJ, Bailey ST, Krakow SL, Minami C, Ishii S, Tanaka H \& Lazar MA 1998 A potent antidiabetic thiazolidinedione with unique peroxisome proliferator-activated receptor $\gamma$-activating properties. Journal of Biological Chemistry 273 32679-32684. (doi:10.1074/jbc.273.49.32679)

Ren D, Collingwood TN, Rebar EJ, Wolffe AP \& Camp HS 2002 PPAR $\gamma$ knockdown by engineered transcription factors: exogenous PPAR $\gamma 2$ but not PPAR $\gamma 1$ reactivates adipogenesis. Genes and Development 16 27-32. (doi:10.1101/gad.953802)

Sadie-Van Gijsen H, Crowther NJ, Hough FS \& Ferris WF 2010 Depotspecific differences in the insulin response of adipose-derived stromal cells. Molecular and Cellular Endocrinology 328 22-27. (doi:10.1016/j. mce.2010.06.009)

Sakamoto J, Kimura H, Moriyama S, Odaka H, Momose Y, Sugiyama Y \& Sawada H 2000 Activation of human peroxisome proliferator-activated receptor (PPAR) subtypes by pioglitazone. Biochemical and Biophysical Research Communications 278 704-711. (doi:10.1006/bbrc.2000.3868)

Satokata I, Ma L, Ohshima H, Bei M, Woo I, Nishizawa K, Maeda T, Takano Y, Uchiyama M, Heaney S et al. 2000 Msx2 deficiency in mice causes pleiotropic defects in bone growth and ectodermal organ formation. Nature Genetics 24 391-395. (doi:10.1038/74231)

Sewter C, Blows F, Considine R, Vidal-Puig A \& O'Rahilly S 2002 Differential effects of adiposity on peroxisomal proliferator-activated receptor $\gamma 1$ and $\gamma 2$ messenger ribonucleic acid expression in human adipocytes. Journal of Clinical Endocrinology and Metabolism 87 42034207. (doi:10.1210/jc.2002-011511)

Shockley KR, Rosen CJ, Churchill GA \& Lecka-Czernik B 2007 PPAR $\gamma 2$ regulates a molecular signature of marrow mesenchymal stem cells. PPAR Research 2007 81219. (doi:10.1155/2007/81219)

Solomon DH, Cadarette SM, Choudhry NK, Canning C, Levin R \& Stürmer T 2009 A cohort study of thiazolidinediones and fractures in older adults with diabetes. Journal of Clinical Endocrinology and Metabolism 94 2792-2798. (doi:10.1210/jc.2008-2157)

Sottile V \& Seuwen K 2000 Bone morphogenetic protein-2 stimulates adipogenic differentiation of mesenchymal precursor cells in synergy with BRL 49653 (rosiglitazone). FEBS Letters 475 201-204. (doi:10.1016/S0014-5793(00)01655-0)

Tanabe Y, Koga M, Saito M, Matsunaga Y \& Nakayama K 2004 Inhibition of adipocyte differentiation by mechanical stretching through ERKmediated downregulation of PPAR $\gamma 2$. Journal of Cell Science $\mathbf{1 1 7}$ 3605-3614. (doi:10.1242/jcs.01207)

Upton R, Widdowson PS, Ishii S, Tanaka H \& Williams G 1998 Improved metabolic status and insulin sensitivity in obese fatty (fa/fa) Zucker rats and Zucker Diabetic Fatty (ZDF) rats treated with the thiazolidinedione, MCC-555. British Journal of Pharmacology 125 1708-1714. (doi:10.1038/ sj.bjp.0702245)

Van Dieren S, Czernichow S, Chalmers J, Kengne AP, de Galan BE, Poulter N, Woodward M, Beulens JWJ, Grobbee DE, van der Schouw YT et al. 2012 Weight changes and their predictors amongst 11140 patients with type 2 diabetes in the ADVANCE trial. Diabetes, Obesity and Metabolism 14 464-469. (doi:10.1111/j.1463-1326.2012.01556.x)

Van Wijngaarden P, Brereton HM, Coster DJ \& Williams KA 2007 Stability of housekeeping gene expression in the rat retina during exposure to cyclic hyperoxia. Molecular Vision 13 1508-1515. 
Wang L, Li L, Gao H \& Li Y 2012 Effect of pioglitazone on transdifferentiation of preosteoblasts from rat bone mesenchymal stem cells into adipocytes. Journal of Huazhong University of Science and Technology. Medical Sciences 32 530-533. (doi:10.1007/s11596-0120091-x)

Yau H, Rivera K, Lomonaco R \& Cusi K 2013 The future of thiazolidinedione therapy in the management of type 2 diabetes mellitus. Current Diabetes Reports 13 329-341. (doi:10.1007/ s11892-013-0378-8)

Zhu Y, Qi C, Korenberg JR, Chen XN, Noya D, Rao MS \& Reddy JK 1995 Structural organization of mouse peroxisome proliferator-activated receptor $\gamma(\operatorname{mPPAR} \gamma)$ gene: alternative promoter use and different splicing yield two mPPAR $\gamma$ isoforms. PNAS 92 7921-7925. (doi:10.1073/pnas.92.17.7921)

Received in final form 26 August 2014

Accepted 10 September 2014

Accepted Preprint published online 10 September 2014
Published by Bioscientifica Ltd. 